Anadolu Üniversitesi Bilim ve Teknoloji Dergisi A-Uygulamalı Bilimler ve Mühendislik Anadolu University Journal of Science and Technology A- Applied Sciences and Engineering Cilt: 16 Sayı: 12015

Sayfa: $23-37$

DOI: $10.18038 /$ btd-a.93366

ARASTIRMA MAKALES / RESEARCH ARTICLE

Sevil ŞENTÜRK ${ }^{1}$

\author{
BULANIK REGRESYON KONTROL GRAF Ğ N N BULANIK MOD VE BULANIK \\ MEDYAN DÖNÜŞÜM TEKN KLER KULLANILARAK OLUŞTURULMASI VE B R \\ UYGULAMA
}

\title{
ÖZ
}

statistiksel süreç kontrolünde eğer sürecin ortalamasında ve değişkenliğinde kararlı bir artma veya azalma gözlemleniyorsa ve süreçte bir aşınma söz konusuysa regresyon kontrol grafiğgi kullanılabilecek en uygun kontrol grafiği tekniğidir. Bununla birlikte, klasik kontrol grafikleri süreçten alınan verilerin kesin ve tam olarak bilindiği durumlarda kullanılmaktadır. lgilenilen süreç, ölçüm sisteminden veya çevresel etkenlerden kaynaklanan bir belirsizlik içeriyorsa bulanık küme teorisine dayalı, bulanık kontrol grafikleri süreci modellemede daha etkin bir araç olarak kullanılabilmektedir. Bulanık regresyon kontrol grafiği, verilerin yaklaşık değer olarak ya da dilsel olarak ifade edildiği ve ortalamanın bir trende sahip olduğu süreçleri değerlendirmede kullanılan bir kontrol grafiğidir. Şentürk (2010) tarafından bulanık regresyon kontrol grafiğinin ve bulanık değişim aralığı kontrol grafiğinin teorik yapısı oluşturulmuş ve üçgen bulanık sayılar için $\alpha$-kesim bulanık orta değişim tekniği kullanılarak uygulaması yapılmıştır. Bu çalışmada ise, yamuk bulanık sayılar kullanılarak, bulanık mod ve bulanık medyan transformasyon teknikleri için bulanık regresyon kontrol grafiğinin ve bulanık değişim aralığı kontrol grafiğinin teorik yapısı ilk defa oluşturulmuştur. Bulanık regresyon kontrol grafiği ve bulanık değişim aralı̆̆ kontrol grafiği için bulanık medyan tekniğine dayalı bir uygulama yapılmış ve uygulama sonuçları yorumlanmıştır.

Anahtar Kelimler: Bulanık dönüşüm teknikleri, Yamuk bulanık sayılar, Kontrol grafikleri, Regresyon kontrol grafiği.

\section{GENERATING FUZZY REGRESSION CONTROL CHART BY USING FUZZY MOD AND FUZZY MEDIAN TRANSFORMATION TECHNIQUES AND AN APPLICATION}

\section{ABSTRACT}

In statistical process control, if there is a consistent increase or decrease in process average and variation and there is a tool wear in process, the regression control chart is the most suitable control chart. Therefore, classical control chart is used in the situation that the data is precise. In case that the monitoring process includes vagueness which arises from measurement system or environmental conditions, fuzzy control charts based on fuzzy set theory are useful tools used to model process. The fuzzy regression control chart is a functional technique to evaluate the process in which the avarege has a trend and data represents a linguistic or aprroximate value. The theoratical structure of fuzzy regression control chart and fuzzy range control chart was generated by Şentürk (2010) and an application was carried out for triangular fuzzy number by using $\alpha$-cut fuzzy midrange technique. In this paper, the theoretical structure of fuzzy regression control chart and fuzzy range control chart are generated for fuzzy mode and fuzzy median by using trapezoidal fuzzy numbers for the first time. An application based on fuzzy median technique carried out for fuzzy regression control chart and fuzzy range control chart. The results are interpreted.

Keywords: : Fuzzy transformation techniques, Trapezoidal fuzzy numbers, Control charts, Regression control chart.

\footnotetext{
1, Anadolu Üniversitesi, Fen Fakültesi statistik Bölümü.

E-posta: sdeligoz@anadolu.edu.tr
}

Geliş: 3 Aralık 2014 Düzeltme: 05 Mayıs 2015 Kabul: 21 Mayıs 2015 


\section{G R Ş}

Dünyada hızla gelişen teknoloji ve gitgide mükemmele yaklaşma isteği ve belki de doğanın bir gün aynısının yapay yollarla ortaya çıkarılması arzusu yapay zeka, yapay sinir ağları ve bulanık mantık kavramlarını gündeme getirmektedir. Bulanık mantık, temelde çok değerli mantık, olasılık kuramı ve yapay zeka alanları üzerine oturtturulmuş bir kavramdır. Yapay zeka araçlarından biri olan bulanık mantık kavramı, her gün kullandığımız ve davranışlarımızı yorumladığımız bir sisteme ulaşmamızı sağlayan matematiksel bir disiplin olarak karşımıza çıkmaktadır (Şenol, 2000).

Bulanık mantık, incelenen olayın çok karmaşık olması ve bununla ilgili yeterli bilginin bulunmaması durumunda kişilerin görüş ve değer yargılarına yer verilmesine ihtiyaç gösteren durumlarda geçerli olmaktadır. Belirsizlik anlamına gelen bulanık mantık bu prensipten yola çıkarak kesin olmayan bir bilgiyi basit ve kullanışlı bir hale getirmektedir (Timothy, 1995). Bulanık mantık yaklaşımı, bulanık küme teorisi üzerine kurulmuş bir yaklaşımdır. Bulanık mantık tam olmayan bilgiyi ve uzman sistemlerdeki yaklaşık yargılamayı elde etmek için kullanılmaktadır. Esas olarak insan düşünme ve algılarındaki belirsizliklerle ilgilenmekte ve bu belirsizliği sayısallaştırmaya çalışmaktadır. Bir başka ifadeyle, bulanık mantık "insanların tam ve kesin olmayan bilgiler ışığında tutarlı ve doğru kararlar vermelerini sağlayan düşünme ve karar mekanizmalarının modellenmesi” olarak tanımlanabilmektedir (Türkbey, 2003).

Bulanık mantık yönteminin uygulanabilirliği bulanık küme teorisine dayanmaktadır. Bulanık bir A kümesini aşağıdaki şekilde ifade etmek mümkün olmaktadır:

$\mathrm{X}$ boş olmayan bir küme olmak üzere; $\mathrm{X}^{\prime}$ deki bir bulanık A kümesi

$$
\forall \mathrm{x} \in \mathrm{Xiçin} \mathrm{;} \mu_{\mathrm{A}}(\mathrm{x}): \mathrm{X} \rightarrow[0,1]
$$

olarak ifade edilebilmektedir. Burada $\mu_{A}(x)$ 'e, bulanık kümeye karşılık gelen üyelik fonksiyonu adı verilmektedir. $\mu_{A}(x)$; A'nın elemanlarının istenilen özelliği hangi ölçüde sağladığının ifadesi olmaktadır. Bulanık küme teorisinde bir eleman bir kümeye 0 ve 1 dahil olmak üzere, 0 ile 1 arasında değişen üyelik dereceleri ile ait olmaktadır. Başka bir deyişle bulanık kümelerde bir bulanık küme elemanı bir kümeye biraz aittir veya biraz değildir denilebilmektedir (Klir ve Yuan,1995; Zadeh,1965). Bulanık kümelerde kullanılan bulanık mantık üyelik fonksiyonları denetlenen sürecin özelliklerine göre uygulamalarda en çok kullanılan fonksiyonlar olan, Uçgen üyelik fonksiyonu, Yamuk üyelik fonksiyonu, Gauss üyelik fonksiyonu ve Genelleştirilmiş Bell üyelik fonksiyonu olarak karşımıza ç1kabilmektedir.

1965 yılında Zadeh (1965) tarafından bulanık küme teorisinin ortaya çıkmasıyla bulanık küme teorisi ile bilinen istatistiksel yöntemler, yapılan çalışmalarda birlikte kullanılmaya başlanmıştır. Bulanık mantık ve istatistiksel yöntemler birbirini tamamlayıcı yöntemler olarak da ifade edilmektedir. Bulanık mantıkta istatistiksel yöntemlerin kullanıldığı çalışmalar "bulanık istatistik" (fuzzy statistics) olarak adlandırılmaktadır (Taheri, 2003). Bulanık mantık teknikleri istatistikte, deney planlamasında, zaman serileri analizinde, regresyon çözümlemesinde, olasılık teorisinde, konjoint analizinde, parametrik ve parametrik olmayan hipotez testlerinde, kalite kontrolünde ve birçok istatistiksel çalışmada kullanılmaktadır.

Literatürde bulanık kontrol grafiklerini konu alan birçok çalışma bulunmaktadır. Bulanık kalite kontrol grafikleri ile ilgili ilk çalışmayı Wang ve Raz (1990) niteliksel kontrol grafikleri için gerçekleştirmiştir. Raz ve Wang (1990) diğer çalışmalarında niteliksel olasılıksal yaklaşım ve üyelik yaklaşımı olarak iki yöntem geliş̧irerek p kontrol grafiği ile uygulamışlardır. Temelleri Wang ve Raz tarafından ortaya atılan bulanık niteliksel kontrol grafikleri için farklı yaklaşımlarla (Kanagawa vd,1993; Rowlands ve Wang,1990; El-Shal ve Morris,2000; Hsu ve Chen 2001; Gülbay vd.2004; Cheng,2005; Gülbay ve Kahraman,2006a ve 2006b, Şentürk vd.,2011; Erginel,2014) literatürde çalışmalar mevcuttur. 
Niceliksel bulanık kontrol grafiklerinin ilki ise Faraz ve Moghadam (2007) çalışmasıdır. Bu çalışmada Shewhart in ortalama kontrol grafiğgine alternatif bir yöntem geliştirmişlerdir. Önerdikleri yöntemin süreçteki kaymaları da daha net bir şekilde ortaya koyduğunu görmüşlerdir. Bulanık Niceliksel kontrol grafiklerine ilişkin $\alpha$-kesim ( $\alpha$-cut) yaklaşımı kullanılarak yapılan ilk çalışma ise ortalama ve değişim aralığı kontrol grafikleri için Şentürk ve Erginel (2009) tarafından literatüre kazandırılmıştır. Daha sonra Erginel vd. (2011) yeni bir yaklaşımla bulanık ortalama ve standart sapma kontrol grafiğini oluşturmuşlardır. Ayrıca Erginel (2011) niceliksel kontrol grafiklerinden birimler kontrol grafiği için, bulanık birimler kontrol grafiğinin teorik yapısını oluşturarak literatüre katkıda bulunmuştur. Niceliksel kontrol grafiklerinden biri olan regresyon kontrol grafiği için bulanık regresyon kontrol grafiği ile ilgili ilk çalışma ise Şentürk (2010) tarafından gerçekleştirilmiştir. Şentürk çalışmasında bulanık küme kısımlarından $\alpha$-kesim yöntemini kullanarak bulanık regresyon kontrol grafiğinin ve bulanık değişim aralığı kontrol grafiğinin üçgen ve yamuk bulanık sayılar için teorik yapısını oluşturmuş̧ur. Üçgen bulanık sayılar için $\alpha$-kesim bulanık orta değişim ( $\alpha$-cut fuzzy midrange) tekniğini kullanarak alet aşınması problemine bulanık regresyon kontrol grafiğini ilk defa uygulamıştır.

$\mathrm{Bu}$ çalışmanın amacı ise, bulanık regresyon kontrol grafiği ve bulanık değişim aralı̆̆ kontrol grafiğinin teorik yapısını bulanık mod ve bulanık medyan dönüşüm tekniklerini kullanarak oluşturmaktır. Bu amaçla yamuk bulanık sayılar kullanılarak, bulanık mod (fuzzy mode) ve bulanık medyan (fuzzy median) dönüşüm tekniği için bulanık regresyon kontrol grafiğinin ve bulanık değişim aralığ 1 kontrol grafiğinin sınırları ilk defa bu çalışmada oluşturulmuştur. Belirtici istatistiksel bir teknik olan medyanın kendi özelliği olan serideki aşır değerlerden etkilenmeme durumunu göz önüne alarak bu çalışmada bulanık regresyon kontrol grafiği ve bulanık değissim aralığı kontrol grafiğgi için bulanık medyan tekniğine dayalı bir uygulama gerçekleştirilmiştir. Yonga levha yoğunluk değerleri için uygulama yapılmış ve uygulama sonuçları yorumlanmıştır.

\section{BULANIK REGRESYON KONTROL GRAF Ğ}

statistiksel süreç kontrolü ( SK) bir sürecin olağan biçimde devam edip etmediğinin istatistiksel yöntemlerle kontrolü olarak tanımlanmaktadır. statistiksel süreç kontrolü ile sürecin olağan bir biçimde devam edip-etmediği istatistiksel yöntemlerle sınanırken, olağan dışı bir durum varsa bunun fark edilip giderilmesi amaçlanır. SK'nın bir diğer amacı da kalite karakteristiklerindeki değişkenliği sistematik olarak azaltmaktır (Burnak,1997). Kaliteyi ve üretimi geliştirmede ve süreci izlemede kullanılan SK'nın temel aracı ise kontrol grafikleridir.

Kontrol grafikleri 1920 yılında W.A. Shewhart tarafından ortaya atılmıştır. Shewhart'in kontrol grafikleri olarak adlandırılan kontrol grafikleri sürecin ilgilenilen karakteristiğine göre niceliksel kontrol grafikleri ve niteliksel kontrol grafikleri olmak üzere iki kategoride incelenmektedir. Niceliksel kontrol grafiklerinde sürecin ilgilenilen karakteristiği ölçümlenebilen bir değer iken, niteliksel kontrol grafiklerinde sürecin ilgilenilen karakteristiği ölçülemeyen fakat sayılabilen bir değer olmaktadır. Niceliksel kontrol grafikleri ortalama ve değişim aralığ kontrol grafiği $(\bar{X}-R)$ ve ortalama ve standart sapma kontrol grafiği $(\bar{X}-S)$ olarak sinıflandırılırken, niteliksel kontrol grafikleri ise $\mathrm{p}, \mathrm{np}, \mathrm{c}$ ve u kontrol grafiği olarak karşımıza çıkmaktadır (Montgomery, 1991).

Niceliksel ölçüler için geliştirilen kontrol grafikleri uygulamalarda bazı durumlarda yetersiz kalarak süreçteki sapmalar karşı duyarlı olamamışlardır. Bu sebeple karşılaşılan sorunları çözebilmek adına niceliksel ölçüler için birçok kontrol grafiği geliştirilmiştir. Söz konusu kontrol grafikleri; değişim katsayısı kontrol grafiği, birimler kontrol grafiğ i, medyan kontrol grafiği, hareketli ortalamalar kontrol grafiği ve regresyon kontrol grafiğidir.

Regresyon kontrol grafiği sürecin ortalaması düzenli bir artış ya da azalış gösterdiğinde yani örneklerden türetilen göstergeler genel bir eğilime sahip olduğunda kullanılan kontrol grafiğidir. Çünkü sürecin ortalaması düzenli bir artı̧̧ ya da azalış gösterdiğinde ortalama kontrol grafiği yetersiz kalmaktadır. Klasik regresyon kontrol grafiğinin gösterimi Şekil 1 de verilmiştir. 


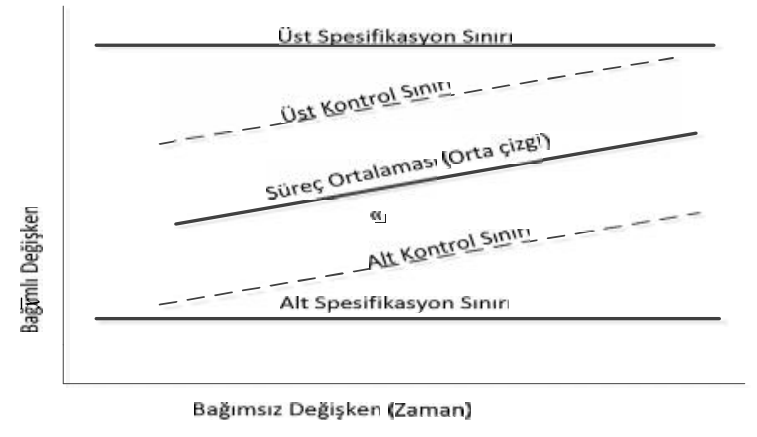

Şekil 1. Klasik Regresyon Kontrol Grafiği Gösterimi

Örneğin, kesme ve delme işlemlerinde kullanılan aletlerin aşınmaya uğraması durumunda ya da kullanılan malzemenin durağan yaşlanma özelliğine sahip olmaması durumunda süreç ortalaması sözü edilen eğilimi gösterebilir ve ilgilenilen kalite karakteristiği zamana bağlı olarak azalabilir ya da artabilir (Burnak,1997). Bu durumda sürecin ortalamasını tahmin etmede en küçük kareler yönteminden yararlanılır. Klasik regresyon kontrol grafiklerinin alet aşınması problemine uygulanması Mandel (1969) tarafından gerçekleştirilmiştir. Kalite karakteristikleri için Mandelin tanımladığı doğrusal regresyon modeli $\left(\bar{x}_{j}=\beta_{0}+\beta_{1} t_{j}+\varepsilon\right)$ şeklindedir. Doğrusal regresyon modelinin parametreleri $\left(\beta_{0}, \beta_{1}\right)$ normal denklemlerin çözümünü temel alan en küçük kareler metodu ile tahmin edilmektedir.

$$
\begin{aligned}
& \sum_{j=1}^{m} \bar{x}_{j}=m \beta_{0}+\beta_{1} \sum_{j=1}^{m} t_{j} \\
& \sum_{j=1}^{m} \bar{x}_{j} \cdot t_{j}=\beta_{0} \sum_{j=1}^{m} t_{j}+\beta_{1} \sum_{j=1}^{m} t_{j}^{2}
\end{aligned}
$$

Söz konusu normal denklemlerde $\bar{x}_{j}=\bar{X}_{j}-\overline{\bar{X}}$ ile hesaplanır. Burada $\bar{X}_{j}$, n örneklem büyüklüğündeki j. örneklemin ortalamasıdır. $\overline{\bar{X}}$ ise genel ortalama olarak ifade edilmektedir. $t_{j}$ ise j. örneklemin numarasıdır $(j=1,2, \cdots, m)$. Normal denklemlerin çözümüyle tahmin değerleri $\left(\hat{\beta_{0}}, \hat{\beta_{1}}\right)$ aşağıda verilen eşitliklerle hesaplanır.

$$
\begin{gathered}
\hat{\beta_{0}}=\frac{\sum_{j=1}^{m} \bar{x}_{j}-\hat{\beta_{1}} \sum_{j=1}^{m} t_{j}}{m} \\
\hat{\beta_{1}}=\frac{\sum_{j=1}^{m} \bar{x}_{j}\left(t_{j}-\bar{t}\right)}{\sum_{j=1}^{m}\left(t_{j}-\bar{t}\right)^{2}}
\end{gathered}
$$

Verilen bilgilerle klasik regresyon kontrol grafiğinin sınırları ise,

$$
\begin{aligned}
& \ddot{U} K S_{\operatorname{Re} g-\bar{X}, j}=\hat{\beta_{0}}+\hat{\beta_{1}} t_{j}+A_{2} \bar{R} \\
& O C_{\operatorname{Re} g-\bar{X}, j}=\hat{\beta_{0}}+\hat{\beta_{1}} t_{j} \\
& A K S_{\operatorname{Re} g-\bar{X}, j}=\hat{\beta_{0}}+\hat{\beta_{1}} t_{j}-A_{2} \bar{R}
\end{aligned}
$$

şeklinde belirlenmektedir. Burada $A_{2}$ sabit katsayı iken, $\bar{R}$ ise değişim aralıklarının ortalamasıdır. 
Regresyon kontrol grafiği ile birlikte sürecin değişkenliğinde meydana gelen değiş̧iminde değişim aralığı kontrol grafiği ile izlenmesi gerekmektedir. Klasik değişim aralığı kontrol grafiğinin sınırları ise;

$$
\begin{aligned}
& \ddot{U} K S_{R}=D_{4} \bar{R} \\
& O C_{R}=\bar{R} \\
& A K S_{R}=D_{3} \bar{R}
\end{aligned}
$$

şeklinde hesaplanmaktadır. Burada $D_{3}$ ve $D_{4}$ örneklem büyüklüğüne bağlı sabit katsayılardır. (Burnak,1997, Montgomery,1991)

Sürecin ortalamasında meydana gelen düzenli artış ve azalışı, dalgalanmaları ve kaymaları yakalamak ise süreci daha erken kontrol altına almak açısından ve maliyetleri azaltmak açısından oldukça önemlidir. Süreçteki bir sorunun mümkün olduğunca erken tespit edilmesi, kontrol dişı bir durum varsa nedenlerinin araştırılması ve sürecin kontrol altına alınarak gerekli önlemlerin alınması ise kalite kontrolünde oldukça önem teşkil etmektedir. Bahsedilen söz konusu durumların süreçte kolaylıkla gözlenebilmesi ve süreci daha iyi yansitan kontrol grafiklerinin oluşturabilmesi için klasik kontrol grafikleri yerine bulanık kontrol grafiklerinin kullanımı daha yararlı sonuçlar vermektedir. Klasik kontrol grafikleri verilerin kesin ve tam olarak bilindiği durumlarda kullanıma uygundur. Eğer ilgilenilen süreç, ölçüm sisteminden veya çevresel etkenlerden kaynaklanan bir belirsizlik içeriyorsa bulanık küme teorisi ve bulanık küme teorisine dayalı bulanık kontrol grafikleri süreci modellemede daha etkin bir araç olarak kullanılabilmektedir.

Bulanık regresyon kontrol grafiği de belirsizlik ortamında süreç verilerini analiz etmede kullanılabilecek etkin bir kontrol grafiğidir. Bulanık regresyon kontrol grafiğinin teorik yapısı bulanık dönüşüm teknikleri ile elde edilebilmektedir. Bulanık mod, $\alpha$-kesim bulanık orta değişim ve bulanık medyan olmak üzere üç dönüşüm tekniği bulunmaktadır. Bulanık dönüşüm tekniklerin den hangisinin kullanılacağının seçimi konusunda ise herhangi bir teorik temel bulunmamaktadır. (Wang ve Raz,1990). Bulanık dönüşüm teknikleri bulanık sayıları kesin sayılara dönüştürmek için kullanılan tekniklerdir. Bu çalışmada bulanık yamuk sayılar kullanılarak bulanık regresyon kontrol grafiği oluşturulacaktır. Bir bulanık yamuk sayı ise $(a, b, c, d)$ olmak üzere dört parametre ile özelleşmiştir. Söz konusu üyelik fonksiyonunun denklemi ise,

$$
\operatorname{yamuk}(x ; a, b, c, d)=\left\{\begin{array}{cc}
0, & x<a . \\
\frac{x-a}{b-a} & a \leq x \leq b . \\
1, & b \leq x \leq c . \\
\frac{d-x}{d-c} & c \leq x \leq d . \\
0, & d<x .
\end{array}\right\}
$$

şeklinde ifade edilmektedir.

Şentürk (2010) tarafından ilk defa teorik yapısı oluşturulan bulanık regresyon kontrol grafiğinde, bulanık yamuk sayılar $\left(X_{a, i j}, X_{b, i j}, X_{c, i j}, X_{d, i j}\right)$ olarak ifade edilirse her bir örneğin bulanık ortalaması $\left(\bar{X}_{a, j}, \bar{X}_{b, j}, \bar{X}_{c, j}, \bar{X}_{d, j}\right)$ olacaktır. Hesaplamaları ise eşitliklerde verildiği gibidir:

$$
\bar{X}_{a, j}=\frac{\sum_{i=1}^{n} X_{a, i j}}{n} \quad \bar{X}_{b, j}=\frac{\sum_{i=1}^{n} X_{b, i j}}{n} \quad \bar{X}_{c, j}=\frac{\sum_{i=1}^{n} X_{c, i j}}{n} \quad \bar{X}_{d, j}=\frac{\sum_{i=1}^{n} X_{d, i j}}{n}
$$

Burada $i=1,2, \ldots, n$ ve $j=1,2, \ldots, m$. 
Her bir bulanık yamuk sayının ortalamasına ilişkin bulanık regresyon modelleri ise aşağıda verilen eşitliklerle tahmin edilecektir. Denklemlerdeki $\left(\beta_{0}, \beta_{1}\right)$ değerlerinin hesaplanışı ise eşitlik 4 ve 5 de verildiği gibidir.

$$
\begin{aligned}
& \bar{X}_{\operatorname{Re} g-a, j}=\hat{\beta}_{0 a}+\hat{\beta}_{1 a} t_{j}+\varepsilon \\
& \bar{X}_{\operatorname{Re} g-b, j}=\hat{\beta}_{0 b}+\hat{\beta}_{1 b} t_{j}+\varepsilon \\
& \bar{X}_{\operatorname{Re} g-c, j}=\hat{\beta}_{0 c}+\hat{\beta}_{1 c} t_{j}+\varepsilon \\
& \bar{X}_{\operatorname{Re} g-d, j}=\hat{\beta}_{0 d}+\hat{\beta}_{1 d} t_{j}+\varepsilon
\end{aligned}
$$

Bulanık yamuk sayılara ilişkin genel ortalamaların hesaplanması ise

$$
\overline{\bar{X}}_{a}=\frac{\sum_{j=1}^{m} \bar{X}_{a, j}}{m} \quad \overline{\bar{X}}_{b}=\frac{\sum_{j=1}^{m} \bar{X}_{b, j}}{m} \quad \overline{\bar{X}}_{c}=\frac{\sum_{j=1}^{m} \bar{X}_{c, j}}{m} \quad \overline{\bar{X}}_{d}=\frac{\sum_{j=1}^{m} \bar{X}_{d, j}}{m}
$$

şeklindedir. Ayrıca $R_{a, j}, R_{b, j}, R_{c, j}, R_{d, j}$ her bir örneğin yamuk değişim aralığı değeri olmak üzere

$$
\begin{aligned}
& R_{a, j}=X_{e n b, a, j}-X_{e n k, d, j} \\
& R_{b, j}=X_{e n b, b, j}-X_{e n k, c, j} \\
& R_{c, j}=X_{e n b, c, j}-X_{e n k, b, j} \\
& R_{c, j}=X_{e n b, d, j}-X_{e n k, a, j}
\end{aligned}
$$

değerleri elde edilir ve yamuk değişim aralıklarının ortalaması ise verilen eşitlik deki gibi hesaplanır.

$$
\bar{R}_{a}=\frac{\sum_{j=1}^{m} R_{a, j}}{m} \quad \bar{R}_{b}=\frac{\sum_{j=1}^{m} R_{b, j}}{m} \quad \bar{R}_{c}=\frac{\sum_{j=1}^{m} R_{c, j}}{m} \quad \bar{R}_{d}=\frac{\sum_{j=1}^{m} R_{d, j}}{m}
$$

Böylece yapılan hesaplamalarla yamuk sayılar için bulanık $\tilde{\bar{X}}$ regresyon kontrol grafiğinin sınırları elde edilir.

$$
\begin{aligned}
& \ddot{U} \tilde{K} S_{\operatorname{Re} g-\bar{X}, j}=\left(\hat{\beta_{0 a}}+\hat{\beta_{1 a}} t_{j}+A_{2} \bar{R}_{a}, \hat{\beta_{0 b}}+\hat{\beta_{1 b}} t_{j}+A_{2} \bar{R}_{b}, \hat{\beta_{0 c}}+\hat{\beta_{1 c}} t_{j}+A_{2} \bar{R}_{c}, \hat{\beta_{0 d}}+\hat{\beta_{1 d}} t_{j}+A_{2} \bar{R}_{d}\right) \\
& =\left(\ddot{U} \tilde{K} S_{1}, \ddot{U} \tilde{K} S_{2}, \ddot{U} \tilde{K} S_{3}, \ddot{U} \tilde{K} S_{4}\right) \\
& O \tilde{C}_{\operatorname{Re} g-\bar{X}, j}=\left(\hat{\beta_{0 a}}+\hat{\beta_{1 a}} t_{j}, \hat{\beta_{0 b}}+\hat{\beta_{1 b}} t_{j}, \hat{\beta_{0 c}}+\hat{\beta_{1 c}} t_{j}, \hat{\beta_{0 d}}+\hat{\beta_{1 d}} t_{j}\right) \\
& =\left(O \tilde{C}_{1}, O \tilde{C}_{2}, O \tilde{C}_{3}, O \tilde{C}_{4}\right) \\
& A \tilde{K} S_{\operatorname{Re} g-\bar{X}, j}=\left(\hat{\beta_{0 a}}+\hat{\beta_{1 a}} t_{j}-A_{2} \bar{R}_{d}, \hat{\beta_{0 b}}+\hat{\beta}_{1 b} t_{j}-A_{2} \bar{R}_{c}, \hat{\beta_{0 c}}+\hat{\beta_{1 c}} t_{j}-A_{2} \bar{R}_{b}, \hat{\beta_{0 d}}+\hat{\beta_{1 d}} t_{j}-A_{2} \bar{R}_{a}\right) \\
& =\left(A \tilde{K} S_{1}, A \tilde{K} S_{2}, A \tilde{K} S_{3}, A \tilde{K} S_{4}\right)
\end{aligned}
$$

Yamuk sayılar için bulanık değişim aralığı kontrol grafiğinin sınırları ise; 


$$
\begin{aligned}
& \ddot{U} \tilde{K} S_{R}=D_{4}\left(\bar{R}_{a}, \bar{R}_{b}, \bar{R}_{c}, \bar{R}_{d}\right)=\left(D_{4} \bar{R}_{a}, D_{4} \bar{R}_{b}, D_{4} \bar{R}_{c}, D_{4} \bar{R}_{d}\right)=\left(\ddot{U} \tilde{K} S_{R_{1}}, \ddot{U} \tilde{K} S_{R_{2}}, \ddot{U} \tilde{K} S_{R_{3}}, \ddot{U} \tilde{K} S_{R_{4}}\right) \\
& O \tilde{C}_{R}=\left(\bar{R}_{a}, \bar{R}_{b}, \bar{R}_{c}, \bar{R}_{d}\right)=\left(O \tilde{C}_{R_{1}}, O \tilde{C}_{R_{2}}, O \tilde{C}_{R_{3}}, O \tilde{C}_{R_{4}}\right) \\
& A \tilde{K} S_{R}=D_{3}\left(\bar{R}_{a}, \bar{R}_{b}, \bar{R}_{c}, \bar{R}_{d}\right)=\left(D_{3} \bar{R}_{a}, D_{3} \bar{R}_{b}, D_{3} \bar{R}_{c}, D_{3} \bar{R}_{d}\right)=\left(A \tilde{K} S_{R_{1}}, A \tilde{K} S_{R_{2}}, A \tilde{K} S_{R_{3}}, A \tilde{K} S_{R_{4}}\right)
\end{aligned}
$$

şeklinde hesaplanır (Şentürk,2010).

Bulanık yamuk sayılar için bulanık regresyon kontrol grafiğgi ve bulanık değişim aralığı kontrol grafiğinin teorik yapısı verildikten sonra söz konusu kontrol grafiklerini bulanık mod ve bulanık medyan dönüşüm tekniklerine entegre ederek karar kurallarını oluşturmak mümkündür. Şentürk (2010) çalışmasında $\alpha$-kesim bulanık orta değişim dönüşüm tekniğine dayalı bulanık regresyon kontrol grafiği ve bulanık değişim aralığı kontrol grafiğine ilişkin karar kurallarını oluşturmuştur. Ancak bu çalışmada bulanık mod ve bulanık medyan dönüşüm teknikleri kontrol grafiklerine entegre edilerek karar kuralları oluşturulmuştur. zleyen alt bölümlerde söz konusu teorik yapı verilmiştir.

\subsection{Bulanık Mod Dönüşüm Tekniği ile Bulanık Regresyon Kontrol Grafiği}

Bulanık mod, $f_{\text {mode }}$ olarak gösterilmektedir. F bulanık küme olmak üzere, F'in bulanık mod değeri, üyelik fonksiyonu 1'e eşit olan bulanık sayının değeridir.

Eşitlik de verildiği gibi hesaplanmaktadır (Wang ve raz,1990):

$$
f_{\text {mode }}=\left\{x \mid \mu_{\mathrm{F}}(x)=1\right\}, \quad \forall x \in F .
$$

Bulanık regresyon kontrol grafiğini ve bulanık değişim aralığı kontrol grafiğini bulanık mod dönüşüm tekniğini kullanarak dönüştürdüğümüz kontrol grafiği sınırları ise;

$$
\begin{array}{r}
S_{\bmod , j}=\left\lfloor b_{j}, c_{j}\right\rfloor \\
\ddot{U} \tilde{K} S_{\text {mod }}=\left[\ddot{U} \tilde{K} S_{2}, \ddot{U} \tilde{K} S_{3}\right] \\
O \tilde{C ̧}_{\bmod }=\left[O \tilde{C}_{2}, O \tilde{C}_{3}\right] \\
A \tilde{K} S_{\bmod }=\left[A \tilde{K} S_{2}, A \tilde{K} S_{3}\right]
\end{array}
$$

şeklindedir. Bulanık regresyon kontrol grafiğinde, Bulanık mod dönüşüm tekniğini kontrol sınırlarında karar aşamasında kullanabilmek için $\beta_{j}$ değerinin tanımlanması gerekir. $\beta_{j}$ ilgili kontrol grafiğinin sınırları ve örneklem değerleri kullanılarak elde edilen bir yüzde orandır (Gülbay ve Kahraman, 2006a, 2006b).

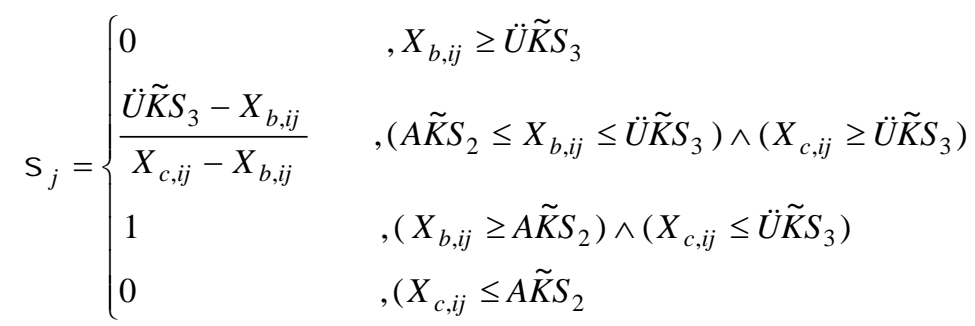

$\beta_{j}$ değeri daha önceden süreç için belirlenen $\beta$ değeri ile karşılaştırılarak sürecin kontrol durumuna aşağıda verilen eşitlik ile karar verilir (Gülbay ve Kahraman, 2006a, 2006b). 
Süreç Kontrol $= \begin{cases}\text { kontrol altında } & , \beta=1\left(X_{b, i j} \geq A \tilde{K} S_{2} \wedge X_{\mathrm{c}, i j} \leq \ddot{U} \tilde{K} S_{3}\right) \\ \text { süreç kontrol dışında } & , \beta=0\left(X_{b, i j} \geq \ddot{U} \tilde{K} S_{3} \vee X_{c, i j} \leq A \tilde{K} S_{s}\right) \\ \text { kısmen kontrol altında } & , \beta_{j} \geq \beta \\ \text { kısmen kontrol dışında } & , \beta_{j}<\beta\end{cases}$

Bulanık değişim aralığı kontrol grafiği içinde $\beta_{j}$ değerinin hesabı

$$
\beta_{j}= \begin{cases}0 & , R_{b, j} \geq \ddot{U} \tilde{K} S_{R_{3}} \\ \frac{\ddot{U} \tilde{K} S_{R_{3}}-R_{b, j}}{R_{c, j}-R_{b, j}} & ,\left(A \tilde{K} S_{R_{2}} \leq R_{b, j} \leq \ddot{U} \tilde{K} S_{R_{3}}\right) \wedge\left(R_{c, j} \geq \ddot{U} \tilde{K} S_{R_{3}}\right) \\ 1 & ,\left(R_{b, j} \geq A \tilde{K} S_{R_{2}}\right) \wedge\left(R_{c, j} \leq \ddot{U} \tilde{K} S_{R_{3}}\right) \\ 0 & ,\left(R_{c, j} \leq A \tilde{K} S_{R_{2}}\right.\end{cases}
$$

ve karar durumu

Süreç Kontrol $= \begin{cases}\text { kontrol altında } & , \beta=1\left(R_{b, j} \geq A \tilde{K} S_{R_{2}} \wedge R_{\mathrm{c}, \mathrm{j}} \leq \ddot{U} \tilde{K} S_{R_{3}}\right) \\ \text { süreç kontrol dışında } & , \beta=0\left(R_{b, j} \geq \ddot{U} \tilde{K} S_{R_{3}} \wedge R_{\mathrm{c}, \mathrm{j}} \leq A \tilde{K} S_{R_{2}}\right) \\ \text { klsmen kontrol altında } & , \beta_{j} \geq \beta \\ \text { klsmen kontrol dışında } & , \beta_{j}<\beta\end{cases}$

hesaplanabilmektedir.

\subsection{Bulanık Medyan Dönüşüm Tekniği ile Bulanık Regresyon Kontrol Grafiği}

Bulanık medyan, $f_{\text {med }}$ olarak ifade edilmektedir. $f_{\text {med }}$ değeri, bulanık kümenin üyelik fonksiyonu altındaki eğriyi iki eşit alana bölen değerdir.

$$
\int_{a}^{f_{\mathrm{med}}} \mu_{F}(x) d x=\int_{f_{\text {med }}}^{d} \mu_{F}(x) d x=\frac{1}{2} \int_{a}^{d} \mu_{F}(x) d x
$$

ve burada a ve $\mathrm{b}$, F bulanık kümesinin temel değişkeninin bitim noktalarıdır. $\alpha$-kesim bulanık medyan değeri ise $f_{\text {med }}^{\alpha}$ ile ifade edilir (Wang ve raz,1990). j. örneklemin $\alpha$-kesim bulanık medyan değeri $S_{m e d, j}^{\alpha}$ ile gösterilirse

$$
S_{m e d, j}^{\alpha}=\frac{1}{4}\left(a_{j}^{\alpha}+b_{j}+c_{j}+d_{j}^{\alpha}\right)
$$

eşitliği ile hesaplanır. Bulanık medyan dönüşüm tekniği ile bulanık $\tilde{\bar{X}}$-regresyon kontrol grafiğinin sinırları ise,

$$
\begin{aligned}
& \ddot{U} K S_{m e d-\operatorname{Re} g-\bar{X}, j}^{\alpha}=O C_{m e d-\operatorname{Re} g-\bar{X}, j}^{\alpha}+A_{2}\left(\frac{\bar{R}_{a}^{\alpha}+\bar{R}_{d}^{\alpha}}{2}\right) \\
& O C_{m e d-\operatorname{Re} g-\bar{X}, j}^{\alpha}=f_{m e d-\operatorname{Re} g-\bar{X}, j}^{\alpha}(O \tilde{C})=\frac{1}{4}\left(\bar{X}_{\operatorname{Re} g-a, j}^{\alpha}+\bar{X}_{\operatorname{Re} g-b, j}+\bar{X}_{\operatorname{Re} g-c, j}+\bar{X}_{\operatorname{Re} g-d, j}^{\alpha}\right) \\
& A K S_{m e d-\operatorname{Re} g-\bar{X}, j}^{\alpha}=O C_{\text {med-Re } g-\bar{X}, j}^{\alpha}-A_{2}\left(\frac{\bar{R}_{a}^{\alpha}+\bar{R}_{d}^{\alpha}}{2}\right)
\end{aligned}
$$


ifadeleri ile oluşturulur. $S_{m e d-\operatorname{Re} g-\bar{X}, j}^{\alpha}$ değerinin bulanık regresyon kontrol grafiği için değeri elde edildikten sonra karar kuralı oluşturulmaktadır.

$S_{m e d-\operatorname{Re} g-\bar{X}, j}^{\alpha}=\frac{1}{4}\left(\bar{X}_{\operatorname{Re} g-a, j}^{\alpha}+\bar{X}_{\operatorname{Re} g-b, j}+\bar{X}_{\operatorname{Re} g-c, j}+\bar{X}_{\operatorname{Re} g-d, j}^{\alpha}\right)$

Bulanık $\tilde{\bar{X}}$-regresyon kontrol grafiğinin sınırlarına dayanarak oluşturulan karar kuralı ise aşağıda verildiği gibidir.

Süreç Kontrol $=\left\{\begin{array}{ll}\text { kontrol altında }, & A K S_{\text {med }-x}^{\alpha} \leq S_{\text {med }-\operatorname{Reg}-\bar{X}, j}^{\alpha} \leq \ddot{U} K S_{\text {med-x }}^{\alpha} \\ \text { kontrol dişı } \quad, & \text { d.d }\end{array}\right\}$

Bulanık medyan dönüşüm tekniği ile bulanık $\tilde{\bar{X}}$-regresyon kontrol grafiğinin sınırları oluşturup süreç yorumlanırken sürecin değişkenliğinin de yorumlanması gerektiğinden bulanık değişim aralığı kontrol grafiğinin sınırlanı ise,

$$
\begin{aligned}
& \ddot{U} K S_{\text {med-R }}^{\alpha}=D_{4} f_{\text {med-R }}^{\alpha}(O \tilde{C}) \\
& O C_{\text {med-R }}^{\alpha}=f_{\text {med-R }}^{\alpha}(O \tilde{C})=\frac{1}{4}\left(\bar{R}_{a}^{\alpha}+\bar{R}_{b}+\bar{R}_{c}+\bar{R}_{d}^{\alpha}\right) \\
& A K S_{\text {med-R }}^{\alpha}=D_{3} f_{\text {med-R }}^{\alpha}(O \tilde{C})
\end{aligned}
$$

elde edilir. $S_{m e d-R, j}^{\alpha}$ değeri ile bulanık regresyon kontrol grafiği için karar kuralı oluşturulur.

$S_{m e d-R, j}^{\alpha}=\frac{1}{4}\left(R_{a, j}^{\alpha}+R_{b, j}+R_{c, j}+R_{d, j}^{\alpha}\right)$

Süreç Kontrol $=\left\{\begin{array}{ll}\text { kontrol altında }, & A K S_{\text {med }-\mathrm{R}}^{\alpha} \leq S_{\text {med-R,j}}^{\alpha} \leq \ddot{U} K S_{\text {med-R }}^{\alpha} \\ \text { kontrol dişı } \quad, & \text { d.d }\end{array}\right\}$

Bulanık yamuk sayılar için bulanık regresyon kontrol grafiği ve bulanık değişim aralığı kontrol grafiklerini bulanık mod ve bulanık medyan dönüşüm tekniklerine entegre ederek karar kuralları elde edildikten sonra, söz konusu grafikler için uygulama ise bulanık medyan dönüşüm tekniği ile yapılmıştır.

\section{UYGULAMA: BULANIK REGRESYON KONTROL GRAF $\breve{G}$ LE YONGA LEVHA YOĞUNLUK DEĞERLER N N NCELENMES}

Uygulamada bir üretim sürecinden elde edilen her biri 5 birimden oluşan 10 örnek için ölçülen toplam 50 birimlik yonga levha yoğunluk değeri üzerinde çalış1lmıştır. Süreç ortalamasında meydana gelen kararlı artma eğilimi durumunda regresyon kontrol grafiği kullanılabilecek en uygun kalite kontrol grafiğidir. Sürecin ortalamasında meydana gelen bu kararlı artışın sebebi ise genellikle makine eskimesi ve ya alet aşınmasıdır. Süreç, ölçüm sisteminden veya çevresel etkenlerden kaynaklanan bir belirsizlik içeriyorsa bulanık regresyon kontrol grafiği süreci modellemede kullanılabilmektedir. Süreçten elde edilen zamana bağlı bulanık yonga levha yoğunluk değerlerinin yamuk bulanık sayılarla elde edilişi Tablo 1 de verilmiştir. 
Tablo 1: Yonga levha yoğunluk değerlerine ait 10 örneklemin yamuk bulanık sayı değerleri

\begin{tabular}{|c|c|c|c|c|}
\hline Zaman & $X_{a, i j}$ & $X_{b, i j}$ & $X_{c, i j}$ & $X_{d, i j}$ \\
\hline 1 & 608 & 609.870 & 609.880 & 609.900 \\
\hline & 608 & 608.290 & 608.300 & 608.400 \\
\hline & 639.100 & 639.600 & 639.600 & 639.800 \\
\hline & 601.208 & 601.209 & 601.209 & 602 \\
\hline & 602.350 & 602.449 & 602.450 & 603.121 \\
\hline $\mathbf{2}$ & 625.700 & 625,740 & 625,750 & 626 \\
\hline & 594.102 & 595,530 & 595,540 & 596 \\
\hline & 591 & 592,720 & 592,730 & 593 \\
\hline & 605.010 & 605,030 & 605,040 & 606 \\
\hline & 597.680 & 597,690 & 597,690 & 597,700 \\
\hline$\vdots$ & $\vdots$ & $\vdots$ & $\vdots$ & $\vdots$ \\
\hline $\mathbf{1 0}$ & 649 & 650.200 & 650.200 & 650.289 \\
\hline & 632.490 & 632.580 & 632.590 & 633 \\
\hline & 650.800 & 650.840 & 650.850 & 650.890 \\
\hline & 640.051 & 640.058 & 640.059 & 640.061 \\
\hline & 644.760 & 644.770 & 644.770 & 644.880 \\
\hline
\end{tabular}

lgili verilere ilişkin ortalama yonga levha değerleri (14-17) eşitlikleri ve onların değişim aralığı değerleri ise (19-22) eşitliklerinden hesaplanarak Tablo 2 de verilmiştir.

Ortalama yonga levha yoğunluk değerleri bağımlı değişken ve zaman bağımsız değişken olmak üzere Regresyon denklemleri aşağıdaki gibi edilmiştir.

$$
\begin{aligned}
& \bar{X}_{\operatorname{Re} g-a, j}=606.531+4.1515 t_{j} \\
& \bar{X}_{\operatorname{Re} g-b, j}=607.363+4.0816 t_{j} \\
& \bar{X}_{\operatorname{Re} g-c, j}=607.375+4.0812 t_{j} \\
& \bar{X}_{\operatorname{Re} g-d, j}=607.860+4.0376 t_{j}
\end{aligned}
$$

Tablo 2: Yonga levha yoğunluk değerleri için bulanık aritmetik ortalama ve bulanık değişim aralığı değerleri

\begin{tabular}{|c|c|c|c|c|c|c|c|c|}
\hline Zaman & $\bar{X}_{a, j}$ & $\bar{X}_{b, j}$ & $\bar{X}_{c, j}$ & $\bar{X}_{d, j}$ & $R_{a, j}$ & $R_{b, j}$ & $R_{c, j}$ & $R_{d, j}$ \\
\hline 1 & 611.731 & 612283 & 612.287 & 612.644 & 37.10 & 38.39 & 38.39 & 38.59 \\
\hline 2 & 602.698 & 603.342 & 603.350 & 603.740 & 32.70 & 33.02 & 33.02 & 35 \\
\hline 3 & 629.198 & 629.804 & 629.814 & 630.338 & 77.80 & 79.53 & 79.53 & 79.81 \\
\hline 4 & 623.986 & 624.758 & 624.782 & 625.200 & 13.64 & 14.32 & 14.31 & 15.98 \\
\hline 5 & 629.366 & 630.161 & 630.174 & 630.364 & 27 & 27.37 & 27.39 & 28.10 \\
\hline 6 & 624.756 & 625.355 & 625.366 & 625.502 & 30.12 & 30.46 & 30.44 & 31.04 \\
\hline 7 & 640.752 & 640.886 & 640.904 & 641.016 & 15.84 & 16 & 15.96 & 16.27 \\
\hline 8 & 647.554 & 647.598 & 647.606 & 647.716 & 41.20 & 41.34 & 41.36 & 41.41 \\
\hline 9 & 640.172 & 640.235 & 640.239 & 640.328 & 41.91 & 42.15 & 42.14 & 42.29 \\
\hline 10 & 643.420 & 643.689 & 643.693 & 643.824 & 17.80 & 18.26 & 18.26 & 18.40 \\
\hline
\end{tabular}


Yonga levha yoğunluk değerleri için oluşturulacak olan bulanık regresyon kontrol grafiği için bulanık yoğunluk değerlerinin değişim aralıklarının ortalamasında hesaplanması gerekmektedir. Bulanık yoğunluk değerlerinin değişim aralıkları,

$$
\bar{R}_{a}=33.51 \quad \bar{R}_{b}=34.08 \quad \bar{R}_{c}=34.08 \quad \bar{R}_{d}=34.69
$$

olarak elde edilmiştir. Hesaplanan değerlerle yonga levha yoğunluk değerlerinin bulanık regresyon kontrol grafiği sınırları aşağıdaki gibi elde edilir.

$$
\begin{aligned}
\ddot{U} \tilde{K} S_{\operatorname{Re} g-\bar{X}, j}= & \left(606.531+4.1515 t_{j}+(0.577)(33.51),\right. \\
& 607.363+4.0816 t_{j}+(0.577)(34.08), \\
& 607.375+4.0812 t_{j}+(0.577)(34.08), \\
& \left.607.860+4.0376 t_{j}+(0.577)(34.69)\right) \\
= & \left(625.87+4.1515 t_{j}, 627.03+4.0816 t_{j}, 627.03+4.0812 t_{j}, 627.70+4.0376 t_{j}\right) \\
O \tilde{C}_{\operatorname{Re} g-\bar{X}, j}= & \left(606.531+4.1515 t_{j}, 607.363+4.0816 t_{j}, 607.375+4.0812 t_{j}, 607.860+4.0376 t_{j}\right) \\
A \tilde{K} S_{\operatorname{Re} g-\bar{X}, j}= & \left(606.531+4.1515 t_{j}-(0.577)(34.69),\right. \\
& 607.363+4.0816 t_{j}-(0.577)(34.08), \\
& 607.375+4.0812 t_{j}-(0.577)(34.08), \\
& \left.607.860+4.0376 t_{j}-(0.577)(33.51)\right) \\
= & \left(586.51+4.1515 t_{j}, 587.69+4.0816 t_{j}, 587.70+4.0812 t_{j}, 588.26+4.0376 t_{j}\right)
\end{aligned}
$$

Burada, $\mathrm{n}=5$ için katsayılar tablosundan bakılan tablo değeri $A_{2}=0.577$ olarak alınmıştır (Kolarik.1995).

Bulanık yoğunluk değerlerinin bulanık değim aralığı kontrol grafiği sınırları ise,

$$
\begin{aligned}
& \ddot{U} \tilde{K} S_{R}=2.114(33.51,34.08,34.08,34.69)=(70.84,72.05,72.05,73.33) \\
& O \widetilde{C}_{R}=(33.51,34.08,34.08,34.69) \\
& A \tilde{K} S_{R}=0(33.51,34.08,34.08,34.69)=(0,0,0,0)
\end{aligned}
$$

şeklinde hesaplanır. Burada, $\mathrm{n}=5$ için katsayılar tablosundan elde dilen tablo değerleri $D_{4}=2.114$ ve $D_{3}=0$ dir (Kolarik,1995).

Bulanık medyan dönüşüm tekniğini bulanık regresyon kontrol grafiğine entegre edebilmek için $\alpha$ kesim değerini yamuk bulanık sayılara uygulamak gerekmektedir. $\alpha$-kesim değerini yamuk bulanık sayılara uygulayarak ve bu çalışmada $\alpha=0.75$ alınarak bulanık aritmetik ortalama ve bulanık değişim aralığı ortalama değerlerinin $\alpha$-kesimlerine ulaşılmıştır. Söz konusu hesaplamalar,

$$
\begin{aligned}
& \bar{X}_{\operatorname{Re} g-a, j}^{0.75}=606.1172+2.0234 t_{j} \\
& \bar{X}_{\operatorname{Re} g-d, j}^{0.75}=607.4962+2.0515 t_{j} \\
& \bar{R}_{a}^{0.75}=33.51+0.75(34.08-33.51)=33.94 \\
& \bar{R}_{d}^{0.75}=34.69-0.75(34.69-34.08)=34.23
\end{aligned}
$$


Yonga levha yoğunluk değerleri için bulanık $\tilde{\bar{X}}$ regresyon kontrol grafiğinin sınırlarının $\alpha$-kesim ile edilişi;

$$
\begin{aligned}
\ddot{U} \tilde{K} S_{\operatorname{Re} g-\bar{X}, j}^{0.75}= & \left(606.1172+2.0234 t_{j}+(0.577)(33.94),\right. \\
& 607.363+4.0816 t_{j}+(0.577)(34.08), \\
& 607.375+4.0812 t_{j}+(0.577)(34.08), \\
& \left.607.4962+2.0515 t_{j}+(0.577)(34.23)\right) \\
= & \left(625.7005+2.0234 t_{j}+627.03+4.0816 t_{j}, 627.03+4.0812 t_{j}, 627.3046+2.0515 t_{j}\right) \\
O \tilde{C}_{\operatorname{Re} g-\bar{X}, j}^{0.75}= & \left(606.1172+2.0234 t_{j}, 607.363+4.0816 t_{j}, 607.375+4.0812 t_{j}, 607.4962+2.0515 t_{j}\right) \\
A \tilde{K} S_{\operatorname{Re} g-\bar{X}, j}^{0.75}= & \left(606.1172+2.0234 t_{j}-(0.577)(33.94),\right. \\
& 607.363+4.0816 t_{j}-(0.577)(34.08), \\
& 607.375+4.0812 t_{j}-(0.577)(34.08), \\
& \left.607.4962+2.0515 t_{j}-(0.577)(34.23)\right) \\
= & \left.\left(586.3665+2.0234 t_{j}+587.69+4.0816 t_{j}, 587.70+4.0812 t_{j}, 587.9128+2.0515 t_{j}\right)\right)
\end{aligned}
$$

$\alpha$-kesim bulanık $\tilde{R}$ kontrol grafiğinin sınırlarının değerleri

$$
\begin{aligned}
& \ddot{U} K S_{R}^{0.75}=2.114(33.94,34.08,34.08,34.23)=(71.75,72.05,72.05,72.36) \\
& O C_{R}^{0.75}=(33.94,34.08,34.08,34.23) \\
& A K S_{R}^{0.75}=0(33.94,34.08,34.08,34.23)=(0,0,0,0)
\end{aligned}
$$

şeklinde bulunur.

Bulanık medyan dönüşüm tekniği ile yonga levha yoğunluk değerleri için bulanık $\tilde{\bar{X}}$-regresyon kontrol grafiğginin değerleri eşitliklerde verildiği gibi hesaplanır.

$$
\begin{aligned}
& \ddot{U} K S_{m e d-\operatorname{Re} g-\bar{X}, j}^{0.75}=626.4737+2.0374 t_{j} \\
& O C_{\text {med }-\operatorname{Re} g-\bar{X}, j}^{0.75}=607.0879+3.0594 t_{j} \\
& A K S_{\text {med }-\operatorname{Re} g-\bar{X}, j}^{0.75}=587.1396+2.0374 t_{j}
\end{aligned}
$$

Bulanık medyan dönüşüm tekniği ile bulanık değişim aralığı kontrol grafiği içinde kontrol grafiği sınırlarının oluşturulması gerekmektedir. $\alpha$-kesim bulanık $\widetilde{R}$ kontrol grafiğinin bulanık medyan tekniğine dayalı kontrol sınırları yonga levha yoğunluk değerleri için aşağıdaki gibi elde edilir.

$$
\begin{aligned}
& \ddot{U} K S_{\text {med-R }}^{0.75}=2.114(34.0825)=72.0504 \\
& O C_{\text {med }-R}^{0.75}=34.0825 \\
& A K S_{\text {med-R }}^{\alpha}=0
\end{aligned}
$$


S. Şentürk / Anadolu Üniv. Bilim ve Tek. Der. - A - Uyg. Bil. ve Müh. 16 (1) - 2015

Tablo 3: Bulanık regresyon kontrol grafiği için bulanık medyan kontrol sınırları ve süreç kararı

\begin{tabular}{|c|c|c|c|}
\hline $\mathrm{t}$ & $S_{m e d-\bar{X}, j}^{\alpha}$ & $587.4208+3.0594 t_{j} \leq S_{m e d-\operatorname{Re} g-\bar{X}, j}^{\alpha} \leq 626.7549+3.0594 t_{j}$ & Süreç Kontrol \\
\hline 1 & 610.1473 & $590.4802 \leq S_{m e d-\operatorname{Re} g-\bar{X}, j}^{\alpha} \leq 629.8143$ & Kontrol Altında \\
\hline 2 & 613.2067 & $593.5397 \leq S_{m e d-\operatorname{Re} g-\bar{X}, j}^{\alpha} \leq 632.8737$ & Kontrol Altında \\
\hline 3 & 616.2661 & $596.5991 \leq S_{m e d-\operatorname{Re} g-\bar{X}, j}^{\alpha} \leq 635.9332$ & Kontrol Altında \\
\hline 4 & 619.3256 & $599.6585 \leq S_{m e d-\operatorname{Re} g-\bar{X}, j}^{\alpha} \leq 638.9926$ & Kontrol Altında \\
\hline 5 & 622.3850 & $602.7179 \leq S_{m e d-\operatorname{Re} g-\bar{X}, j}^{\alpha} \leq 642.0520$ & Kontrol Altında \\
\hline 6 & 625.4444 & $605.7774 \leq S_{m e d-\operatorname{Re} g-\bar{X}, j}^{\alpha} \leq 645.1114$ & Kontrol Altında \\
\hline 7 & 628.5038 & $608.8368 \leq S_{m e d-\operatorname{Re} g-\bar{X}, j}^{\alpha} \leq 648.1709$ & Kontrol Altında \\
\hline 8 & 631.5633 & $611.8962 \leq S_{m e d-\operatorname{Re} g-\bar{X}, j}^{\alpha} \leq 651.2303$ & Kontrol Altında \\
\hline 9 & 634.6227 & $614.9556 \leq S_{m e d-\operatorname{Re} g-\bar{X}, j}^{\alpha} \leq 654.2897$ & Kontrol Altında \\
\hline 10 & 637.6821 & $618.0151 \leq S_{m e d-\operatorname{Re} g-\bar{X}, j}^{\alpha} \leq 657.3491$ & Kontrol Altında \\
\hline
\end{tabular}

Tablo 3 incelendiğinde bulanık medyan dönüşüm tekniğine dayalı bulanık regresyon kontrol değerlerine ait tüm yonga levha ortalama yoğunluk değerleri kontrol altındadır.

Tablo 4: Bulanık değişim aralığı kontrol grafiği için bulanık medyan kontrol sınırları ve süreç kararı

\begin{tabular}{|l|l|l|l|}
\hline $\mathbf{t}$ & $S_{\text {med }-R, j}^{\alpha}$ & $0 \leq S_{m e d-R, j}^{\alpha} \leq 72.0504$ & Süreç Kontrol \\
\hline 1 & 38.398 & $0 \leq S_{m e d-R, j}^{\alpha} \leq 72.0504$ & Kontrol Altında \\
\hline 2 & 33.866 & $0 \leq S_{m e d-R, j}^{\alpha} \leq 72.0504$ & Kontrol Altında \\
\hline 3 & 79.544 & $0 \leq S_{m e d-R, j}^{\alpha} \leq 72.0504$ & Kontrol Dişında \\
\hline 4 & 15.003 & $0 \leq S_{m e d-R, j}^{\alpha} \leq 72.0504$ & Kontrol Altında \\
\hline 5 & 27.672 & $0 \leq S_{m e d-R, j}^{\alpha} \leq 72.0504$ & Kontrol Altında \\
\hline 6 & 30.695 & $0 \leq S_{m e d-R, j}^{\alpha} \leq 72.0504$ & Kontrol Altında \\
\hline 7 & 16.106 & $0 \leq S_{m e d-R, j}^{\alpha} \leq 72.0504$ & Kontrol Altında \\
\hline 8 & 41.363 & $0 \leq S_{m e d-R, j}^{\alpha} \leq 72.0504$ & Kontrol Altında \\
\hline 9 & 42.196 & $0 \leq S_{m e d-R, j}^{\alpha} \leq 72.0504$ & Kontrol Altında \\
\hline 10 & 18.293 & $0 \leq S_{m e d-R, j}^{\alpha} \leq 72.0504$ & Kontrol Altında \\
\hline
\end{tabular}


Tablo 4 incelendiğinde ise bulanık medyan dönüşüm tekniğine dayalı bulanık değişim aralığı kontrol değerlerine ait 3.örneklem için bulanık yoğunluk değişim değeri kontrol altında değildir.

\section{SONUÇ VE YORUMLAR}

statistiksel süreç kontrolünde, sürecin ortalamasında ve değişkenliğinde meydana gelen düzenli artış ve azalışı, dalgalanmaları ve kaymaları yakalamak ve süreci daha erken kontrol altına almak oldukça önemlidir. Süreç ortalamasında meydana gelen kararlı artış ve azalışları ortaya koymada ve süreçte bir aşınma söz konusu olduğunda ise regresyon kontrol grafiğini kullanmak uygun olmaktadır. Süreç, ölçüm sisteminden veya çevresel etkenlerden kaynaklanan bir belirsizlik içeriyorsa sürecin modellemesi bulanık regresyon kontrol grafiği ile olmalıdır. Bu çalışmada, bulanık regresyon kontrol grafiği ve bulanık değişim aralığı kontrol grafiğinin teorik yapısı bulanık mod ve bulanık medyan dönüşüm teknikleri kullanarak ilk defa oluşturulmuştur. Uygulamada yonga levha yoğunluk değerleri, bulanık medyan dönüşüm tekniğine dayalı bulanık regresyon kontrol grafiği ve bulanık değişim aralığı kontrol grafiği ile modellenmiştir. Uygulama sonucunda ise yonga levha yoğunluk değerlerine ait sürecin ortalamada kontrol altında iken değişkenlikte kontrol altında olmadı̆̆

\section{TEŞEKKÜR}

Makale; Anadolu Üniversitesi 1505F293 nolu Yayın ve Araştırma Teşvik Projesi ile desteklenmiştir, bu kapsamda teşekkürlerimi bildiririm.

\section{KAYNAKÇA}

Chakravarty, A. K. and Shtub A., (1985)“New Technology Investments in Multistage Production Systems", Decision Science, vol. 16(3), 248-264.

Burnak ,N. (1997). Toplam kalite yönetimi-istatistiksel süreç kontrolü, Osmangazi Üniversitesi Mühendislik Mimarlık Fakültesi, TEKAM yayın No: TS-97-008-NB.

Cheng.,C.B. (2005). Fuzzy Process Control: Construction of Control Charts with Fuzzy Numbers, Fuzzy Sets and Systems, 154 ,287-303.

El-Shal ,S.M. and Morris, A.S. (2000). A Fuzzy Rule-Based Algorithm to Improve The Performance of Statistical Process Control in Quality Systems, Journal of Intelligent Fuzzy Systems, 9 ,207-223.

Erginel, N. (2008). Fuzzy Individual and Moving Range Control Charts with $\alpha$-cuts, Journal of Intelligent Fuzzy Systems, 19, 373-383.

Erginel, N; Şentürk, S; Kahraman, C ve Kaya . (2011). Evaluating the Packing Process in Food Industry using Fuzzy $\tilde{\bar{X}}$ and $\widetilde{\mathrm{S}}$ Control Charts, International Journal of Computational Intelligence Systems, 4, 509-520.

Erginel, N. (2014).Fuzzy Rule Based p-np Control Charts, Journal of Intelligent Fuzzy Systems, 27, 159171.

Faraz A. and Moghadam.M.B. (2007). Fuzzy Control Chart A Beter Alternative for Shewhart Average Chart, Quality \& Quantity, 41,375-385.

Gülbay M. and Kahraman, C.(2006a) Development of Fuzzy Process Control Charts and fuzzy Unnatural Pattern Analyses, Computational Statistics and Data Analysis,51, 434-451.

Gülbay M. and Kahraman.C. (2006b). An Alternative Approach to Fuzzy Control Chart: Direct Fuzzy Approach, Information Sciences,77, 1463-1480.

Gülbay,M; Kahraman C. and Ruan D. (2004). $\alpha$-Cut Fuzzy Control Chart for Linguistic Data, International Journal of Intelligent Systems, 19, 1173-1196. 
Hsu, H.M. and Chen, Y.K. (2001). A Fuzzy Reasoning Based Diagnosis System for $\bar{X}$ Control Charts, Journal of Intelligent Manufacturing,12, 57-64.

Kanagawa, A ;Tamaki, F. and Ohta, H. (1993).Control Charts for Process Average And Variability Based on Linguistic Data, Intelligent Journal of Production Research, 3, 913-922.

Klir, J.G. and Yuan, B. (1995).Fuzzy Sets and Fuzzy Logic Theory and Applications, Prentice Hall , New Jersey.

Kolarik W J. (1995), Creating Quality Concepts, Systems, Strategies and Tools, McGraw Hill.

Mandel, J. (1969). The Regression Control Chart, Journal of Quality Technology, 1 .

Montgomery, D.C. (1991). Introduction to Statistical Quality Control, John Wiley \& Sons. Inc. USA.

Özdamar, .H. (2013).Regrsyon Kontrol Kartı ve Bir Çalışma, Süleyman Demirel Üniversitesi Orman Fakültesi Dergisi, 14, 134-137.

Raz,T. and Wang,J.H. (1990). Probabilistic and memberships approaches in the construction of control chart for linguistic data, Production Planning and Control,1,147-157.

Rowland, H. and Wang, L.R. (2000). An Approach of Fuzzy Logic Evaluation and Control in SPC, Quality Reliability Engineering Intelligent, 16, 91-98.

Şenol, F. (2000). Bulanık Mantık Kontrolcüsü, Gazi Üniversitesi Lisans Tezi, Ankara,

Şentürk,S. and Erginel,N.(2009). Development of fuzzy $\tilde{\bar{X}}-\tilde{R}$ and $\tilde{\bar{X}}-\tilde{S}$ control charts using $\alpha_{-}$ cuts, Information Sciences, 179, 1542-1551.

Şentürk, S, Erginel, N Kaya, and Kahraman, C.(2011).Design of fuzzy $\tilde{u}$ Control charts, Journal of Multiple-Valued Logic and Soft Computing, 17,459-473.

Şentürk, S. (2010). Fuzzy Regression Control Chart Based on $\alpha$-Cut Approximation, International Journal of Computational Intelligence Systems, 3, 123-140.

Taheri, S.M.( 2003).Trends in Fuzzy Statistics, Austrian Journal of Statistics, 32, 239-257.

Timothy, J.R. (1995). Fuzzy Logic with Engineering Applications, Mc Graw-Hill, Newyork,

Türkbey, O. (2003). Makina Sıralama Problemlerinde çok Amaçlı Bulanık Küme Yaklaşımı, Gazi Üniversitesi, Mühendislik. Mimarlık. Fakülte Dergisi, 18, 63-77.

Wang, J.H. and Raz, T. (1990). On The Construction of Control Charts using Linguistic Variables, Intelligent Journal of Production Research, 28, 477-487.

Zadeh, L.A.( 1965).Fuzzy Sets, Information and Control, 8, 338-353. 